\title{
Chapter 15 \\ Nano-Scale Electrical Transducers of Surface Plasmons for Integrated Biosensing
}

\author{
Pieter Neutens, Iwijn De Vlaminck, Sergii Lozenko, Liesbet Lagae, \\ and Pol Van Dorpe
}

\begin{abstract}
Recent developments in fabrication, characterization, and understanding of local surface plasmon resonances and surface plasmon waveguides have fuelled the development of a new generation of surface plasmon based biosensors, mainly based on local refractive index sensing and surface enhanced Raman scattering $[1,2]$. Although the actual sensor has scaled to the nanoscale, the system still requires bulky optical components, such as light sources, lenses, objectives, and detectors. Integrating sources and/or detectors with the plasmonic sensor can pave the way to small-footprint photodetectors. In this chapter, we demonstrate direct electrical detection of surface plasmon resonators and integrated detection and generation of deep-subwavelength guided plasmons in metal-insulator-metal (MIM) waveguides. We will discuss these different devices, including optical/ electrical characterization, the comparison with simulations, and their relevance for integrated biosensors.
\end{abstract}

\section{Electrical Detection of Surface Plasmon Resonance in a Single Plasmonic Resonator}

In the context of biosensing applications, direct electrical transduction of LSPR of a single metal nanostructure using a photodetector in its near field holds great promise. First experimental results for integrated electrical detection of surface plasmons on a relatively large area of randomly distributed nanodisks have been reported [3]. Nevertheless, being able to measure each plasmonic resonator separately has several advantages. First, such a device benefits from a rapid signal reading by an integrated

P. Neutens $\bullet$ I. De Vlaminck • S. Lozenko $\bullet$ L. Lagae • P. Van Dorpe $(\bowtie)$

IMEC, Kapeldreef 75, Leuven 3001, Belgium

e-mail: pvandorp@imec.be 


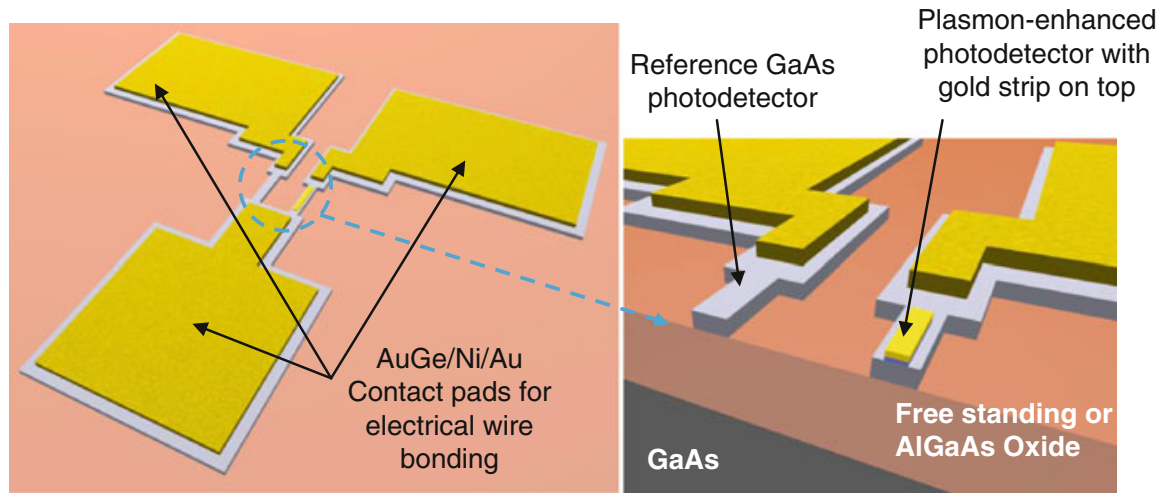

Fig. 15.1 Schematic view of and cross-section of the device comprising reference photodetector and plasmon-enhanced photodetector with a gold strip on top

photodetector. Second, it is compatible with manufacturing technologies used in integrated circuit design. Advancements in nanotechnologies may allow building a large parallel, closely packed array of individual sensing elements on surface plasmon enhanced photodiodes. Thus, the "pixel" size of a sensor is decreased to the size of a single metal nanostructure. Monitoring the signal of single nanostructures also excludes inhomogeneous broadening, which usually occurs when measuring an ensemble of nanoparticles. Such a sensor would allow measurements of the distribution of analyte concentration over the sensor surface as well as perform averaging statistics over large areas. Integration of the sensor arrays together with supporting electronics may lead to development of lab-on-achip systems, uniting all steps of the analytical process in a single device.

It is obvious now, that efficient, small, and fast transducing schemes that can directly convert plasmonic signals into electrical signals are needed for LSPR sensors to fully reveal their potential.

A scheme of local electrical transduction of a plasmon resonance of a single metal nanostructure using a photodetector described here is shown in Fig. 15.1. It is based on a surface plasmon polariton (SPP) resonator coupled to a thin GaAs photoresistor [4]. To take advantage of the confinement of the excited surface plasmons and to avoid electrical shorts, the GaAs photoresistors are fabricated either freestanding, or on top of an electrically and optically insulating oxidized AlGaAs layer.

The optical detector used is a GaAs photoresistor with a thin gold strip fabricated directly on top of it, but separated from the $\mathrm{GaAs}$ by a thin $\mathrm{SiO}_{2}$ layer. A photoresistor detector is chosen mainly because of the ease of fabrication and response interpretation. Close to this detector, a reference detector without the metal structure is constructed. By measuring the device and a reference detector simultaneously, irregularities in the source spectrum and wavelength-dependent absorption characteristics of the GaAs detector can be subtracted from the device response. 
The strip supports surface plasmon modes on top of the strip (at the Au/air interface) and at the bottom of the strip (at the $\mathrm{Au} / \mathrm{SiO}_{2} / \mathrm{GaAs}$ interface). The finite width of the strip results in discrete Fabry-Perot resonances at specific resonance wavelengths. Especially the resonances related to the $\mathrm{Au} / \mathrm{GaAs}$ interface couple strongly to the photodetector and are expected to show up in the spectral photoresponse measurements. Indeed, a comparison of the response of a plasmon-enhanced detector with a gold strip, and a reference detector without the strip reveals a resonance peak in the absorption spectrum at the wavelength corresponding to the excitation of standing wave mode of a SPP at the interface between a gold strip and a semiconducting detector.

Both finite difference time domain (Lumerical FDTD) and finite element (Comsol Multiphysics, RF module) simulations were used to study the response of the surface-plasmon enhanced detector. In Lumerical FDTD, the dielectric properties of the materials were described modeled using a combination of Drude and Lorentz models, which were obtained by curve-fitting experimental data found in literature (GaAs and Au permittivity data taken from Palik and Johnson\&Christy). This works very well except for energies below the GaAs bandgap, as the fitted imaginary part of the permittivity does not turn exactly zero and some "parasitic" absorption remains. A calculation grid size of $2 \mathrm{~nm} \times 2 \mathrm{~nm}$ was chosen for simulations after a convergence analysis.

COMSOL multiphysics [5] implements finite element frequency domain algorithm (FE/FD). Material properties can be described by specifying experimental values of their dielectric constants for each wavelength, which allows a more precise description of GaAs near the bandgap.

\section{Modeling Results}

The purpose of the computational study is to predict the optimal design of the strip-line plasmonic detector, to tailor maximum possible absorption enhancement with the resonance energy lying above to the GaAs band gap.

The geometry of the strip-line plasmonic photodetector (in cross-section) used in the simulations is depicted in Fig. 15.2a. The gold strip on top of the detector is designed as a fragment of a SPP waveguide with sidewalls acting as reflectors, resulting in a Fabry-Perot cavity. The resonant properties of this cavity depend greatly on the dielectric environment and the geometry of the resonator. We assumed that the GaAs photodetector is freestanding.

The relative absorption spectrum of the detector is obtained by monitoring the power flow in and out of the GaAs semiconductor volume and comparing it to the reference detector without the gold strip. In Fig. 15.2b the calculated relative absorption spectrum is presented for different widths of the Au strip. Clearly, strong resonance peaks are observed at $\lambda$ between 700 and $1,000 \mathrm{~nm}$, corresponding to the excitation of a SPP cavity mode. SPPs, excited with TM-polarized light at the edges of the gold strip and propagating on the $\mathrm{Au}-\mathrm{SiO}_{2}-\mathrm{GaAs}$ interface interfere and 
a
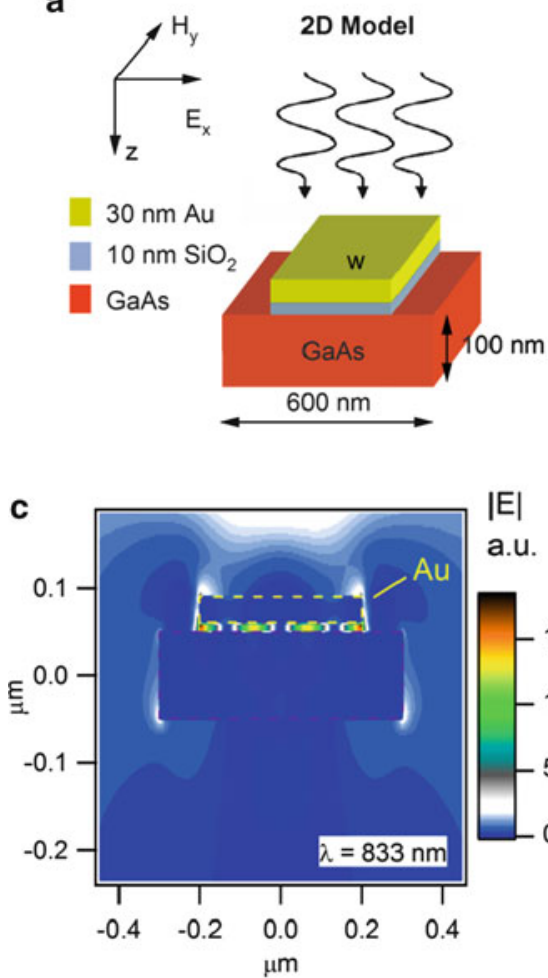

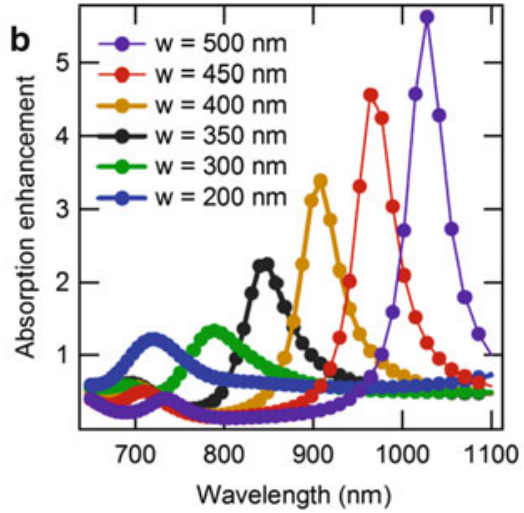

|E $\quad$ d

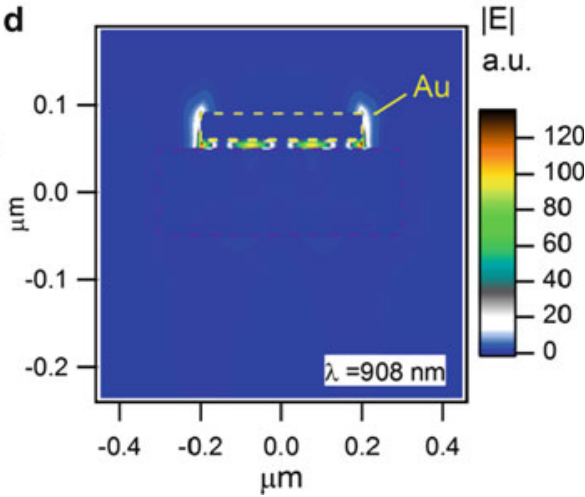

Fig. 15.2 (a) Geometry of the plasmonic detector, used in the simulation. (b) Relative absorption spectrum of the surface plasmon enhanced detector for different widths of the Au strip. (c, d) Electrical field intensity distribution of the surface plasmon polariton (SPP) cavity mode for the off-resonant and resonant excitation wavelengths correspondingly. Note the large difference in electric field enhancement (adapted from [4])

create a standing wave pattern. The electrical field intensity distribution of the SPP mode is visualized for an Au width of $400 \mathrm{~nm}$ in Fig. 15.2 for off-resonant $(\lambda=833 \mathrm{~nm})$ (Fig. 15.2c) and resonant $(\lambda=908 \mathrm{~nm})$ (Fig. 15.2d) excitation wavelengths. The modal profile exhibits four spots of high electric field intensity which corresponds to the establishment of a standing wave pattern with an order number $j=3$. Lower- and higher-order modes can also exist in the same cavity. At the resonant excitation wavelength electric fields of the cavity mode are greatly amplified which leads to enhanced light absorption through transfer of the SPP cavity mode energy to the photodetector. It is evident from the picture that most of the mode energy is confined within the $\mathrm{SiO}_{2}$ spacer layer.

As is clear from Fig. 15.2b, the strength of the resonance and its wavelength depend on the geometry of the cavity: width and thickness of the gold strip, on the efficiency of excitation of the SPP and on the strength of the coupling between the 
Fig. 15.3 Experimental photodetector enhancement for different widths of the Au strip (inset: comparison of the experimental (blue) and simulated (black) resonance wavelength) (adapted from [4])

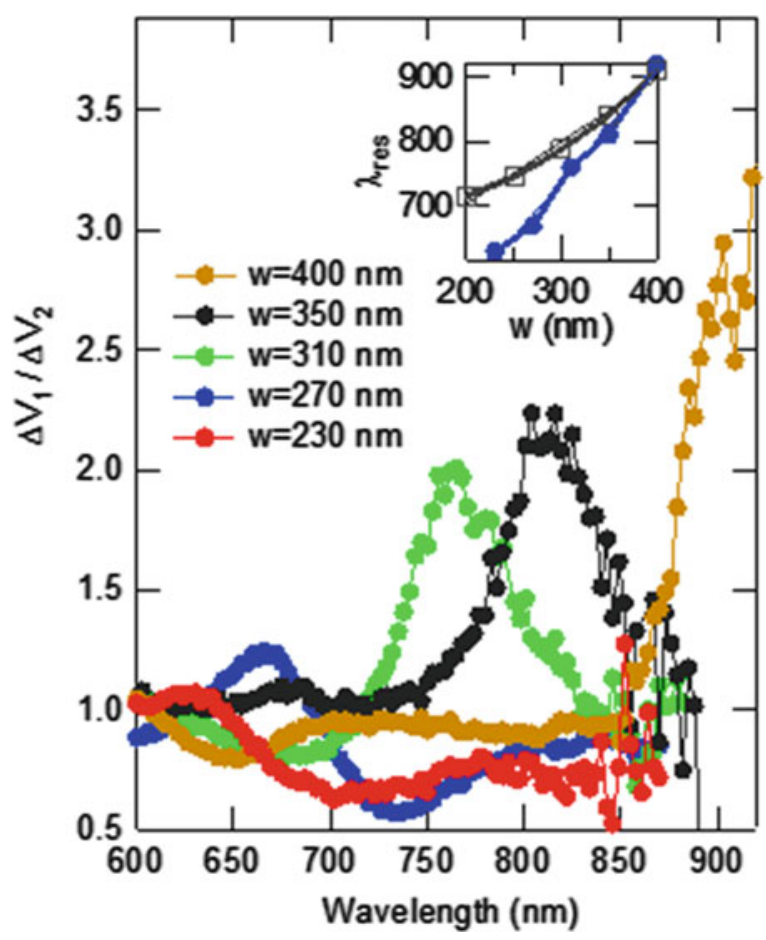

cavity mode and the semiconductor. A wide-range tunability of the resonance can be attained by changing the cavity geometry.

This has been confirmed experimentally [4], as shown in Fig. 15.3, which shows the experimentally obtained photosignal enhancement for different Au strip widths. In the experiment, light from a $300 \mathrm{~W}$ Xe lamp is passed through a monochromator, and is subsequently linearly polarized, modulated with an optical chopper, and focused on the sample with a lens. A device covered with and without the $\mathrm{Au}$ nanostrip is measured simultaneously and their signals are divided to obtain the absorption enhancement. Photosignal enhancements of up to a factor of 3 have been obtained for resonance wavelengths near the GaAs bandgap. For shorter wavelengths, the resonance is strongly damped, as the GaAs absorption strongly increases above the GaAs bandgap.

As for eventual applications, freestanding GaAs wires might not be so appropriate; we also fabricated these GaAs/Au plasmonic detectors on top of an insulating substrate. This has been achieved by oxidizing an underlying $\left(\mathrm{Al}_{0.88}, \mathrm{Ga}_{0.12}\right)$ As layer with a high $\mathrm{Al}$ content, resulting in a transparent AlGaAs oxide, with a refractive index of about 1.65. This is shown in Fig. 15.4a, b, showing the plasmonic and the reference detector.

For this device, clear photosignal resonance signals have been obtained below the GaAs bandgap energy, as illustrated in Fig. 15.4c. 


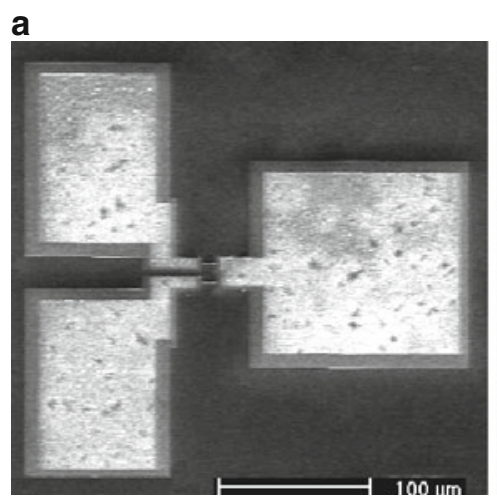

b
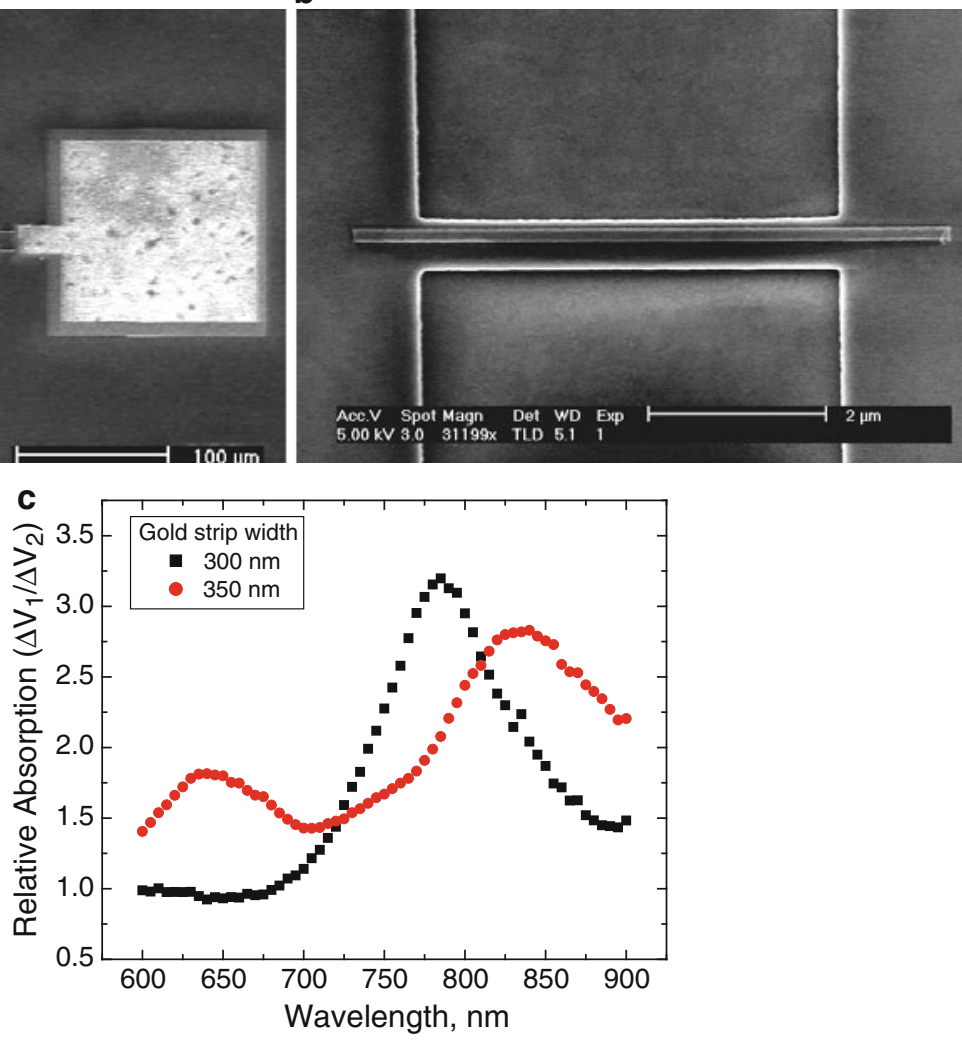

Fig. 15.4 (a) SEM picture of the plasmonic and reference detector fabricated on an insulating substrate. (b) Zoom of the combined Au strip and GaAs photodetector. (c) Optical enhancement spectrum of the plasmonic device for two different widths of the gold strip

\section{Biocompatibility}

To turn the plasmon-enhanced photodetector, described here into a device that can be operated as a biosensor, some design considerations have to be taken into account. One of the main requirements for the biosensors is biocompatibility, which means an ability to work under physiological conditions. The design described above has several disadvantages if to be used for biological sensing: first, exposure of the GaAs semiconducting layer to liquids may lead to the problem of chemical instability; second, charges in the liquid can influence the conductivity of the semiconductor detector. The reference detector should be passivated with a material, transparent in the wavelength range of interest, and the region where liquid and detector interact can be limited by definition of a microfluidic channel. 

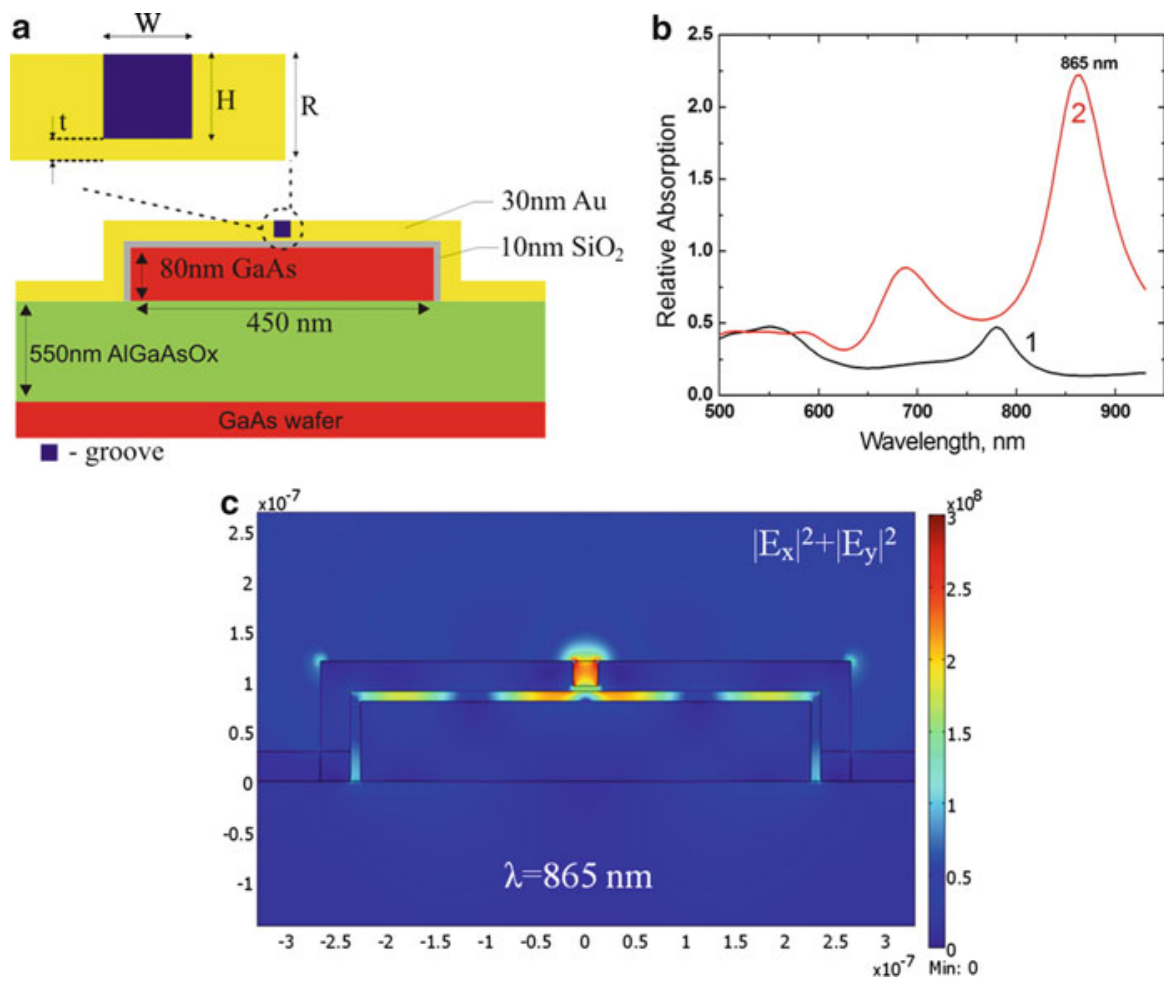

Fig. 15.5 (a) Cross-section view of a nano-groove plasmonic detector. (b) Simulated relative absorption spectra of the detector in the case of full-metal coverage (curve 1), and when a nanogroove is incorporated on the surface (curve 2). (c) Electrical field intensity distribution of the SPP cavity mode at the resonant excitation wavelength

For biological sensing applications it would be desirable to have all the surface of the detector covered with metal, which will on the one hand, protect it from the influence of the environment, solve the electrical instability problem by screening the charges in the liquid and on the other hand, facilitate biofunctionalization. Extending the gold layer all over the detector area seems to be a promising approach, as gold can be easily functionalized. However SPPs are excited mainly on the edges of the gold strip, so the full metal coverage of the detector will dramatically reduce the efficiency of surface plasmon excitation by screening the electromagnetic fields.

A way to achieve a SPP excitation in case when the detector is fully covered with gold is the incorporation of a single corrugation in the form of a nanosized groove on the top of the smooth gold coverage of the detector.

A schematic view of the device concept, used in simulations is presented in Fig.15.5. In Fig. 15.5a, a cross-section of the fully gold-covered plasmonic detector with a nano-groove incorporated on top is shown. 

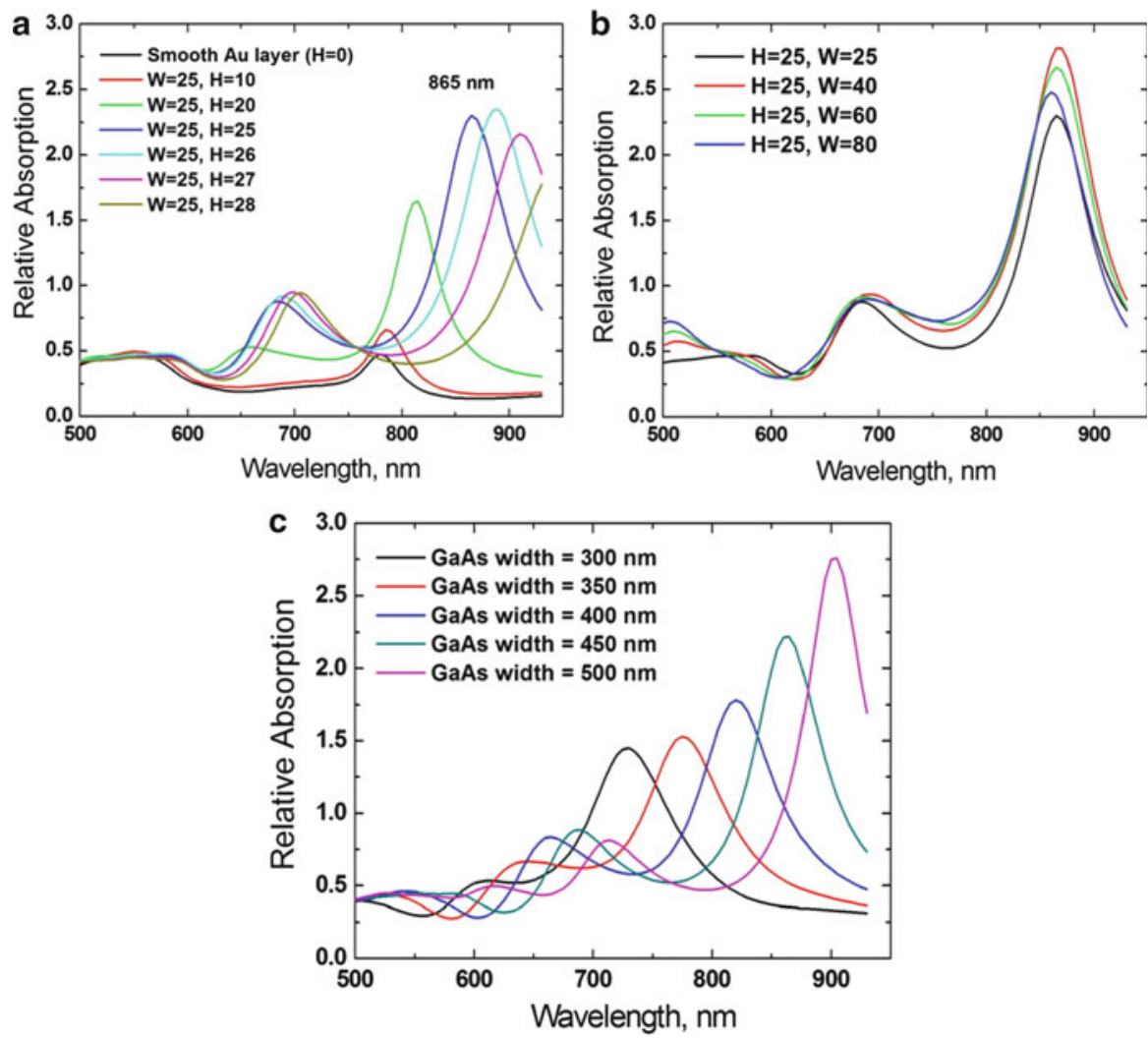

Fig. 15.6 Calculated relative absorption spectra of the nano-groove surface plasmon enhanced detector for a different groove widths and depths. (a) Groove depth is varied in the range 10-28 nm at a constant width of $W=25 \mathrm{~nm}$. (b) Groove width is varied from 25 to $80 \mathrm{~nm}$; depth is $H=25 \mathrm{~nm}$. (c) Photodetector width is varied in the range $300-500 \mathrm{~nm}$

Simulated relative absorption spectra, shown on Fig. 15.5b, confirm that in case when GaAs detector is fully covered with gold layer, efficiency of SPP cavity mode excitation, seen as a small bump on the spectrum (curve 1) is reduced to a great extent. However, if a nanosized groove is incorporated on the otherwise smooth gold surface, efficient surface plasmon excitation and absorption enhancement may be achieved (curve 2).

An excitation through the nano-groove can provide efficient coupling to a $\mathrm{SiO}_{2}$-gold interface SPP mode if the groove profile and gold film thickness are suitably related. In Fig. 15.6 dependence of the relative absorption enhancement on the groove parameters - width $(W)$ and depth $(H)$-is investigated. A GaAs photodetector without the gold layer is taken as a reference in the following calculations.

The simulation results show, that a variation of the groove depth $(H)$ affects the SPP excitation efficiency to a great extent. At a small depth $(H=10 \mathrm{~nm})$, excitation efficiency is very low, resembling the case when no groove is present at the 
surface. As the depth increases, the plasmon excitation efficiency grows up to a certain depth value and resonant peak shifts to the infrared region. The groove depth of $H=25 \mathrm{~nm}$ was found to provide the most efficient coupling of incident radiation to a SPP mode of the order $j=4$ with a corresponding resonant peak at $865 \mathrm{~nm}$ (Fig. 15.6a). The second peak, observed at a lower wavelength is related to the excitation of a higher-order cavity mode $(j=5)$. Groove width variation influences the relative absorption spectra to a less extent, causing the change in relative absorption maximum value and slightly shifting the resonance wavelength. The relative absorption magnitude rises as the groove width increases up to an approximately $40 \mathrm{~nm}$, and drops down again if the groove becomes wider (Fig. 15.6b). Low sensitivity of the surface plasmon excitation efficiency to the groove width gives practical advantages, as very narrow grooves are more difficult to manufacture and small variations in groove width will affect the final device performance to a much less extent.

The resonance peak wavelength position can be tuned, as for an Au-strip plasmonic photodetector, by varying the size of the plasmon cavity. In Fig. $15.5 \mathrm{c}$ the relative absorption spectra are presented as the width of the GaAs photodetector was varied from 300 to $500 \mathrm{~nm}$. As a result of the plasmon cavity length change, a blue-shift of the resonant wavelength is observed together with the decrease of the peak magnitude due to stronger material damping.

\section{Sensitivity}

To evaluate the biosensing capabilities of the described device, the dependence of the response to a change of environmental refractive index above the detector was studied numerically. The nano-groove width was chosen to be $W=50 \mathrm{~nm}$, depth $H=25 \mathrm{~nm}$, and refractive index above the detector was varied in the range from $n=1$ to $n=1.4$ to see the general trends of the device response. Variations of the refractive index due to antibody-antigen interactions in an aqueous environment on the functionalized sensor surface usually lie in the range from 1.3 to 1.4 refractive index units. The corresponding relative absorption spectra are presented in Fig. 15.7a. The resonance peaks corresponding to the quadrupolar $(j=4, \lambda=870 \mathrm{~nm})$ and higher-order $(j=5$, $\lambda=680 \mathrm{~nm}$ ) mode excitation are red-shifted as refractive index of the medium above the detector changes from 1 to 1.4. The relative absorption magnitude varies insignificantly for the quadrupolar resonance peak, however a two times stronger absorption enhancement is achieved for the higher-order mode. The sensitivity of the detector to the refractive index change was estimated to be around $\sim 100 \mathrm{~nm} / \mathrm{RIU}$.

The same calculation was repeated with a totally gold-covered photodetector taken as a reference (Fig. 15.7b). Such an approach highlights the role of the nanogroove in the SPP cavity mode excitation. A drop in the magnitude of relative absorption as refractive index increases may be explained, first by the increase in the absorption efficiency of the reference detector due to the lower index contrast and, most importantly by a reduction of the efficiency of coupling between the surface plasmon mode and the active semiconductor volume. 

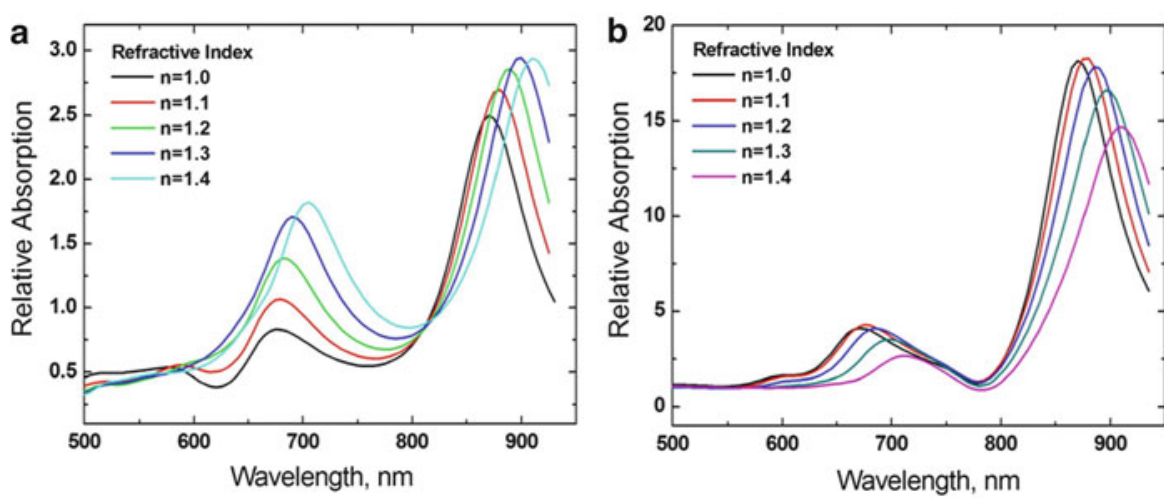

Fig. 15.7 Calculated relative absorption spectra illustrating sensitivity of the nano-groove surface plasmon enhanced detector $(W=50 \mathrm{~nm}, H=25 \mathrm{~nm})$ to refractive index change. (a) GaAs photodetector without metal coverage is used as a reference. (b) Gold-covered photodetector taken as reference

The previously defined "figure of merit" (FOM) will be used to characterize the sensitivity of the proposed sensors. The estimated sensitivity values are summarized in the table below. The obtained sensitivity values are 2-4 times lower than those, found in literature for nanoparticle LSPR sensors [6, 7]. Ways to increase the refractive index sensitivity need to be found for a device to have biosensing capabilities.

\begin{tabular}{llll}
\hline$\lambda_{\text {peak }}, n=1.0(\mathrm{~nm})$ & Sensitivity $(\mathrm{nm} / \mathrm{RIU})$ & FWHM $(\mathrm{nm} / \mathrm{meV})$ & FOM \\
\hline 870 & $\sim 100$ & $\sim 80 / 130$ & 1.2 \\
\hline
\end{tabular}

\section{Advanced Device Design: Nano-Slit Plasmonic Detector}

Since in 1998 Ebbesen has reported on an enhanced light transmission through a thin metal film with subwavelength holes [8], a lot of research has been conducted on the optical properties of nano-structured metallic thin films. It has been shown in Lindberg et al. [9] that the enhanced transmission through a subwavelength slit in a metal film can be understood in terms of interfering waveguide modes that propagate in the slit. The position of the transmission resonance depends strongly on the refractive index of the material in the slit that makes sensing applications possible.

An attempt to excite the SPP cavity resonance of the above described device with the help of the enhanced transmission through the slit in the gold layer was made. The high sensitivity of the enhanced transmission to the refractive index change in the slit, reported in Lindberg et al. [9], may increase the sensing potential of the device. The slit in this case can be treated as a MIM waveguide supporting a propagating TM mode. The lowest order TM mode in the planar metallic waveguide, unlike the TE mode, has no cutoff frequency and exhibits a 

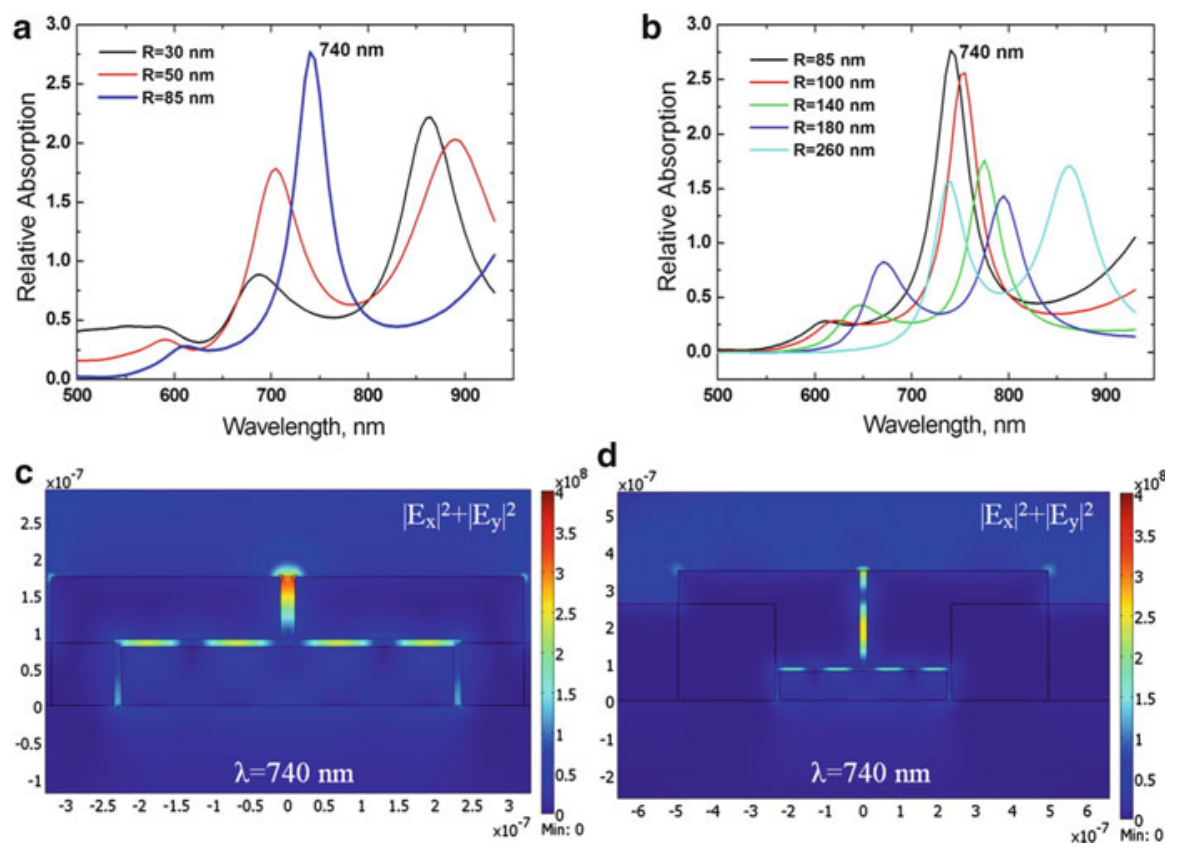

Fig. 15.8 Calculated relative absorption spectra of nano-slit photodetector with $W=20 \mathrm{~nm}$, $t=5 \mathrm{~nm}$. (a) Thickness of the gold layer $R$ is varied from 30 to $85 \mathrm{~nm}$. (b) $R$ is varied from 85 to $180 \mathrm{~nm}$. (c, d) Electrical field intensity distribution of the SPP cavity mode at the resonant excitation wavelength $\lambda=740 \mathrm{~nm}$ for $R=85 \mathrm{~nm}$ and $R=260 \mathrm{~nm}$

surface-wave-like behavior. The aim of the following set of calculations, presented on Fig. 15.8 is to investigate the dependence of SPP cavity resonance of the plasmon enhanced photodetector device on the thickness of the gold layer $(R)$, while keeping groove parameters $W$ and $t$ constant $-t$ is the thickness of the remaining gold layer in the groove, see Fig. 15.5a.

A variation of the gold layer thickness $R$ significantly influences the spectra. As $R$ increases to $85 \mathrm{~nm}$, a maximum in relative absorption is observed at $\lambda=740 \mathrm{~nm}$ that corresponds to the excitation of quadrupolar SPP cavity mode through coupling with the guided mode of MIM slit waveguide. The intensity distribution of the electrical field in the slit shows that strong resonances occur at a certain thickness $R$ when an odd number of the quarters of wavelengths of the TM slit waveguide mode fits into the slit. The wavelength of the slit guided mode is estimated from the intensity distributions to be $\lambda \approx 340 \mathrm{~nm}$. If $R$ is increased to $260 \mathrm{~nm}$, so that three quarters of the wavelength fit into the slit (Fig. 15.8d), the resonance peak is observed at the same wavelength of $\lambda=740 \mathrm{~nm}$ but is almost two times weaker. This can be explained by the strong attenuation of the propagating mode in the slit waveguide in the visible spectral region. 

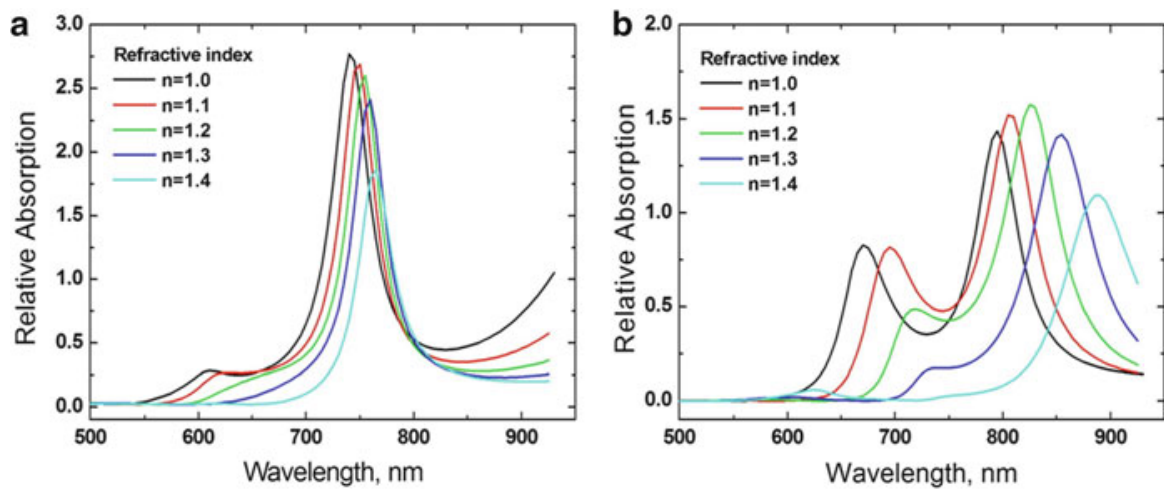

Fig. 15.9 Calculated relative absorption spectra illustrating sensitivity of the nano-slit surface plasmon enhanced detector $(W=20 \mathrm{~nm}, t=5 \mathrm{~nm}$ ) to the refractive index change. (a) Thickness of the gold layer $R=85 \mathrm{~nm}$. (b) Thickness of the gold layer $R=180 \mathrm{~nm}$

The refractive index sensitivity of this device is studied in Fig. 15.9. Calculations have been performed for two values of the gold layer thickness, $R=85 \mathrm{~nm}$ (Fig. 15.8a) and $R=180 \mathrm{~nm}$ (Fig. 15.8b). The slit parameters were chosen to be $W=20 \mathrm{~nm}, t=5 \mathrm{~nm}$. The estimated sensitivity values for the main quadrupolar resonance peak are summarized in the table below.

\begin{tabular}{lllll}
\hline Gold layer thickness $(\mathrm{nm})$ & $\lambda_{\text {peak }}, n=1.0(\mathrm{~nm})$ & Sensitivity $(\mathrm{nm} / \mathrm{RIU})$ & FWHM $(\mathrm{nm} / \mathrm{meV})$ & FOM \\
\hline$R=85$ & 740 & $\sim 62$ & $\sim 50 / 111$ & 1.18 \\
$R=180$ & 795 & $\sim 235$ & $\sim 55 / 110$ & 3.85 \\
\hline
\end{tabular}

Besides the quadrupolar resonance at $\lambda=740 \mathrm{~nm}$, a strong resonance peak corresponding to the excitation of the higher mode of the order $j=5$ is observed in the spectrum $(\lambda=670 \mathrm{~nm})$ when $R=180 \mathrm{~nm}$. The higher-order mode resonance peak is significantly influenced by the change of refractive index in the slit: as index value rises to 1.4 peak completely vanishes. By comparing the signals from the reference and plasmon-enhanced detectors, conclusion can be made that the drop of the peak intensity is due to the change of the refractive index in the slit and therefore, change of the guided mode propagation conditions, as the signal of the reference detector, not covered with gold, stays nearly the same for this wavelengths range.

The obtained FOM values from the nano-slit sensor with $R=180 \mathrm{~nm}$ are comparable with those found in literature for LSPR nanoparticle sensors, making such a device concept a promising candidate for further research. However more deep investigation of the nature of the observed effects and their dependency on geometrical parameters of the nano-slit and the photodetector are needed before proceeding to the practical implementation of the device. 


\section{Electrical Generation and Detection of Surface Plasmon Polaritons in Deep-Subwavelength Surface Plasmon Waveguides}

SPPs, charge density oscillations at metal-dielectric interfaces, have proven to provide excellent means to probe biochemical events due to their strong local field enhancement near metal surfaces or nanostructures. Multiple detection schemes based on surface plasmon resonance (SPR) have already been developed and commercialized [10] to experimentally characterize molecular binding events. It has also been shown that circuits consisting of plasmon waveguides can provide a deep-subwavelength sensing platform. Nevertheless, all these applications however make use of large external light sources and detectors. By combining SPP waveguides and waveguide circuits with integrated electrical SPP sources and detectors, we can integrate plasmonics with today's electronic devices. Doing so, we pave the way for numerous applications, going from integrated plasmonic circuits to arrays of small-scale, fully-integrated plasmonic biosensors.

In this section we focus on demonstrating efficient electrical transduction between plasmonic and electrical signals, by integrating light-emitting-diodes (LED) and metal-semiconductor-metal (MSM) photodetectors directly in deep-subwavelength MIM plasmon waveguides [11, 12], resulting in a scheme which is scalable down to the nanoscale, allowing a fully operational plasmonic circuit on a very small footprint. Plasmonic MIM waveguides offer the prospect of combining a high spatial field confinement together with micrometer range propagation lengths [13, 14]. Metal-based waveguides provide the unique opportunity to send electrical and optical signals through the same guides. Here we exploit this feature by employing the metal slabs for direct electrical contacting of a semiconductor LED and an MSM photodetector structure. We have fabricated such integrated structures on top of GaAs substrates, containing either a p-i-n-diode with three quantum wells (LED) or an undoped GaAs layer (for the MSM detector). The waveguides were fabricated using standard deposition techniques, electron beam lithography, and Xe ion milling. More details regarding the fabrication procedures can be found in Neutens et al. [11, 12].

Figure 15.9 shows both the integration of a detector and a LED in two different devices. Figure 15.9a shows the schematic operation of an integrated detector: Light is focused on a subwavelength slit of the top metal layer of the MIM waveguide. Through the excitation of a local mode in the slit, the waveguide modes of the MIM waveguide can be efficiently excited. Subsequently the propagating MIM mode is captured in a subwavelength slit in the bottom metal layer of the MIM waveguide, where the light is absorbed in the GaAs through the excitation of electron-hole pairs. These carriers are collected by the electrodes, which are formed by etching a slit in the bottom metal layer. Figure 15.9b shows an SEM picture of the processed device, with the excitation slit on the left and the detection slit on the right side indicated by the triangles. Figure 15.9c shows the schematic operation of an integrated LED: by contacting the semiconductor locally below a subwavelength slit in the bottom metal layer and electrically driving the $\mathrm{p}-\mathrm{i}-\mathrm{n}$ diode, excitons are formed in the three 

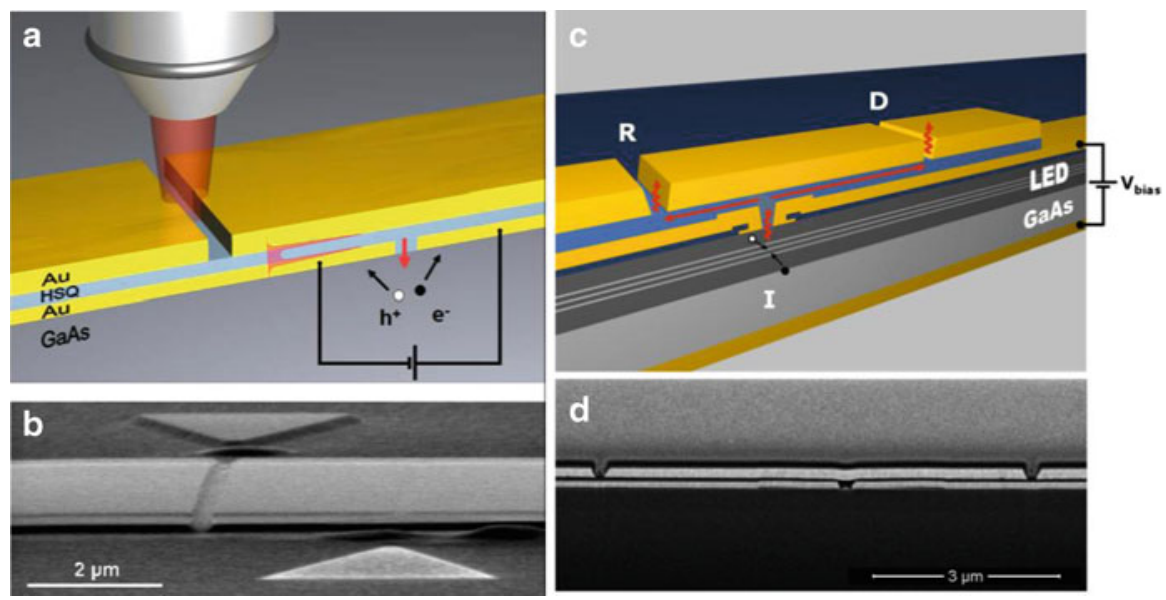

Fig. 15.10 (a) Schematic representation of the MSM detector integrated in a MIM plasmonic waveguide. (b) SEM picture of the final device. (c) Schematic representation of the LED integrated in a MIM plasmonic waveguide. (d) SEM picture of the final device (adapted from $[11,12])$

quantum wells, giving rise to relatively broad-band light emission. The emitted light can directly couple with the subwavelength slit and couple to propagating modes in the MIM waveguide. In order to detect this, a second slit has been etched in the top metal film, which allows coupling of the plasmons to the far-field, where they can be spectrally analyzed and imaged by a CCD camera. Figure 15.9d shows an SEM picture of a processed device.

The operation principle has been modeled using numerical simulations using the RF module of Comsol Multiphysics. For the detector, a plane wave is incident on the slit in the top metal layer of the MIM waveguide. Figure 15.10a shows that for the light polarization oriented perpendicular to the slit (x-direction), efficient coupling between the incident light and MIM waveguide modes is achieved. The observed standing waves in the MIM waveguide originate from reflections at the injection and detection slits. Similarly, we modeled the LED by a point dipole at the position of the quantum wells in the GaAs substrate. Figure 15.10b shows the electric field profile when we put a point dipole with its polarization perpendicular to the slit axis. Also here, an efficient coupling between the dipole and the MIM waveguide modes is observed.

A first indication of surface plasmon propagation in plasmon waveguides is given by the polarization dependence of the measured signals. For the coupled MIM waveguide/detector device, we have mapped the photocurrent of the device as function of the position of the laser spot for two different polarizations (see Fig. 15.11a). It is clear that efficient coupling has only been achieved for the polarization perpendicular to the slit (TM), as expected for plasmonic waveguides. Similarly, we have analyzed the light coupled to the far-field in the LED/waveguide devices. From Fig. 15.11b it is obvious that the light polarization lies perpendicular to the slit axis. 

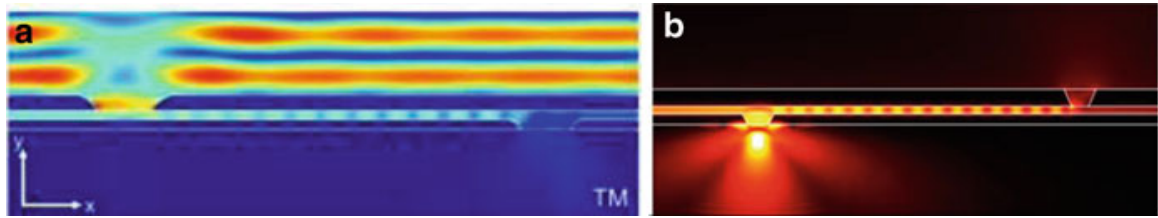

Fig. 15.11 (a) Simulated electric field profile of a plasmonic waveguide coupled to MSM detector. (b) Simulated electric field profile of a LED coupled to a MIM waveguide

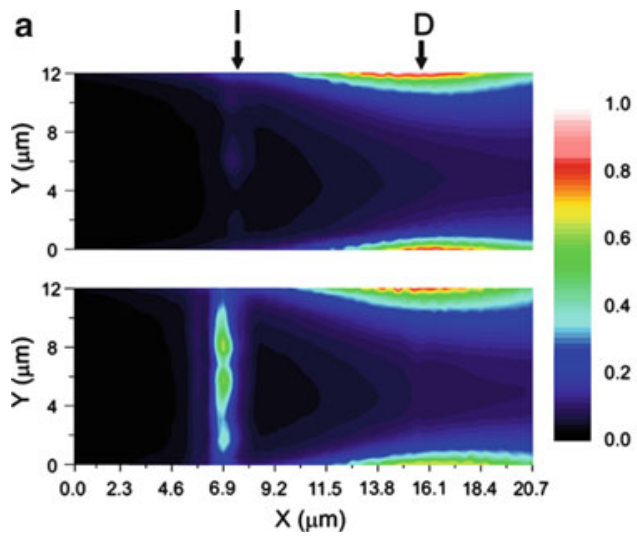

b
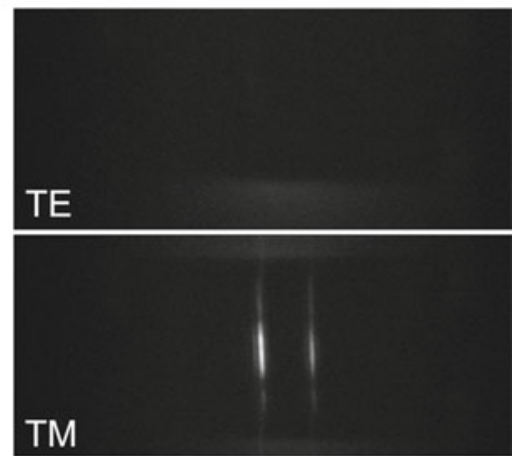

Fig. 15.12 (a) Spatial map of the photocurrent as function of the laser spot position for a polarization parallel (top) and perpendicular to the slit (bottom). The positions of the injection and detection slit are indicated. (b) CCD image of the light emitted through two slits in the top metal layer of the MIM waveguide that is integrated on top of an LED. The polarization of the analyzer was set either parallel to the slit (top) and perpendicular to the slit (bottom)

As surface plasmons decay exponentially along the length of the waveguide due to ohmic losses, this should be manifested in the different devices. Therefore we varied the distance between the injection and detection slits, for both the detector and LED based devices. Figure 15.12a, b indeed shows that the obtained results are consistent with this picture. The obtained plasmon propagation lengths correspond to the simulated results.

This illustrates that we have clearly demonstrated local electrical generation and detection of guided SPPs. The use of such components can be exploited to construct nanoscale SPR-based resonance devices, which can be produced in large arrays, for highly parallelized sensing schemes without the need for external optical components. 

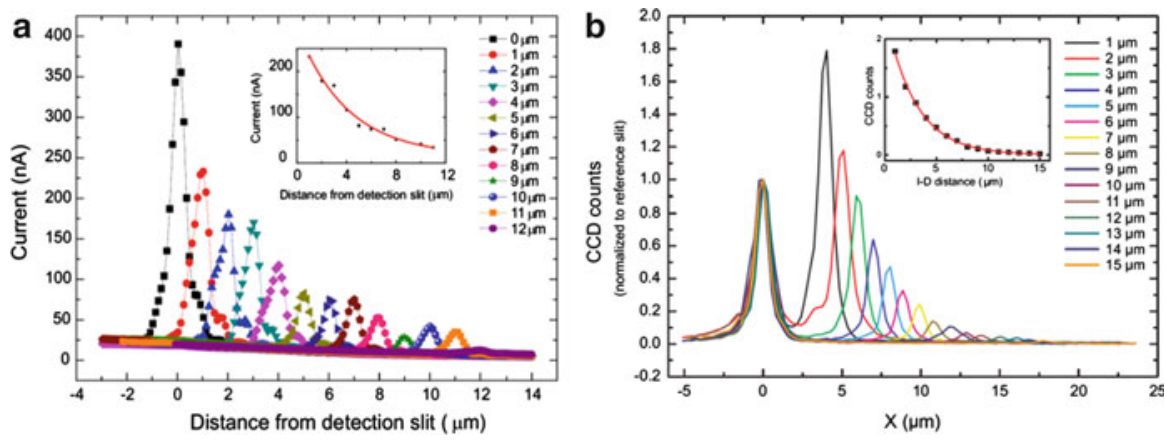

Fig. 15.13 (a) Photocurrent of the MSM photodetectors for different distances between the injection and detection slits. (b) Measured light emission from the slits in the top metal layer for different distances between the injection slit and the out-coupling slit

\section{References}

1. Kneipp K, Wang Y, Kneipp H, Perelman LT, Itzkan I, Dasari RR, Feld MS. Single molecule detection using surface-enhanced Raman scattering (SERS). Phys Rev Lett. 1997;78:1667-70.

2. Haes AJ, Hall WP, Chang L, Klein WL, Van Duyne RP. A localized surface plasmon resonance biosensor: first steps toward an assay for Alzheimer's disease. Nano Lett. 2004;4 (6): 1029-34.

3. Mazzotta F, Wang G, Hägglund C, Höök F, Jonsson MP. Nanoplasmonic biosensing with onchip electrical detection. Biosens Bioelectron. 2010;26(4):1131-6.

4. De Vlaminck I, Van Dorpe P, Lagae L, Borghs G. Local electrical detection of single nanoparticle plasmon resonance. Nano Lett. 2007;7:703-6.

5. www.comsol.com.

6. Sherry LJ, Chang S-H, Wiley BJ, Xia Y, Schatz GC, Van Duyne RP. Localized surface plasmon resonance spectroscopy of single silver nanocubes. Nano Lett. 2005;5(10):2034-8.

7. Sherry LJ, Jin R, Mirkin CA, Schatz GC, Van Duyne RP. Localized surface plasmon resonance spectroscopy of single silver triangular nanoprisms. Nano Lett. 2006;6(9):2060-5.

8. Ebbesen TW, Lezec HJ, Ghaemi HF, Thio T, Wolff PA. Extraordinary optical transmission through sub-wavelength hole arrays. Nature. 1998;391:667-9.

9. Lindberg J, Lindfors K, Setälä T, Kaivola M, Friberg AT. Spectral analysis of resonant transmission of light through a single sub-wavelength slit. Opt Express. 2004;12(4):623-32.

10. www.biacore.com.

11. Neutens P, Van Dorpe P, De Vlaminck I, Lagae L, Borghs G. Electrical detection of confined gap plasmons in metal-insulator-metal waveguides. Nat Photonics. 2009;3:283-6.

12. Neutens P, Van Dorpe P, Lagae L, Borghs G. Electrical excitation of confined surface plasmon polaritons in metallic slot waveguides. Nano Lett. 2010;10:1429-32.

13. Dionne JA, Lezec HJ, Atwater HA. Highly confined photon transport in subwavelength metallic slot waveguides. Nano Lett. 2006;6:1928-32.

14. Dionne JA, Sweatlock LA, Atwater HA, Polman A. Plasmon slot waveguides: towards chipscale propagation with subwavelength-scale localization. Phys Rev B. 2006;73:035407. 